\title{
Aumento dos níveis do receptor solúvel da Interleucina-6 em pacientes com pré-eclâmpsia em relação a gestantes normotensas
}

\author{
Increased levels of the soluble receptor of interleukin-6 in patients with \\ preeclampsia compared to normotensive pregnant women
}

\author{
Marta Ribeiro Hentschke1, Luiza Silveira Lucas², Bruna Krauspenhar³, Raisa Perdomo Felix ${ }^{2}$, \\ Alex Oliboni Sussela ${ }^{2}$, Francine Berlesi ${ }^{2}$, Carlos E. Poli-de-Figueiredo ${ }^{4}$, Bartira E. Pinheiro da Costa ${ }^{5}$ \\ Médica, doutoranda do Programa de Pós-Graduação em Medicina e Ciências da Saúde da Pontifícia Universidade Católica do Rio Grande do Sul \\ (PPGMCS-PUCRS). Porto Alegre, RS. \\ 2 Acadêmicos da Faculdade de Medicina da Pontifícia Universidade Católica do Rio Grande do Sul (FAMED-PUCRS). Porto Alegre, RS. \\ Farmacêutica, mestranda do PPGMCS-PUCRS. Porto Alegre, RS. \\ ${ }^{4}$ Médico Nefrologista. Doutor pela University of Oxford, Inglaterra. Professor Titular do Departamento de Medicina Interna da FAMED-PUCRS \\ e do PPGMCS-PUCRS. Chefe do Serviço de Nefrologia do Hospital São Lucas da PUCRS. Porto Alegre, RS. \\ ${ }^{5}$ Doutora em Ciências pela Universidade Federal do Rio Grande do Sul. Professora Adjunta do Departamento de Medicina Interna da FAMED-PUCRS \\ e do PPGMCS-PUCRS. Coordenadora da Comissão Científica da FAMED-PUCRS. Porto Alegre, RS. \\ Estudo realizado com apoio da Fundação de Amparo à Pesquisa do Rio Grande do Sul (FAPERGS), do Conselho Nacional de Desenvolvimento \\ Cientifico e Tecnológico (CNPq) e da Coordenação de Aperfeiçoamento de Pessoal de Nível Superior (CAPES).
}

\begin{abstract}
RESUMO
Objetivos: Comparar níveis plasmáticos do receptor solúvel da interleucina-6 (IL-6sR) entre gestantes normotensas e com pré-eclâmpsia.

Métodos: Realizou-se coleta de sangue materno no período pré-parto de 21 pacientes com pré-eclâmpsia e 39 controles normotensas. As amostras foram armazenadas a menos $80^{\circ} \mathrm{C}$ até a análise laboratorial. Os níveis séricos de IL-6sR foram mensurados através do teste imunoenzimático ELISA. Para comparar os grupos foi utilizado teste t de Student. Consideraram-se significantes os resultados com P menor do que 0,05 .

Resultados: Os dados de gestantes com pré-eclâmpsia e gestantes normotensas, respectivamente, foram: idade materna $22,3 \pm 4,8$ vs $26,0 \pm 3,7$ anos $(\mathrm{P}=0,06)$; idade gestacional 32,7 $\pm 5,8$ vs $40,1 \pm 0,8$ semanas $(\mathrm{P}=0,01)$; pressão arterial sistólica $143,0 \pm 2,2$ vs $118,8 \pm 3,1 \mathrm{mmHg}(\mathrm{P}=0,01)$; pressão arterial diastólica $112,5 \pm 4,0$ vs $77,2 \pm 10,2 \mathrm{mmHg}(\mathrm{P}=0,01)$; ácido úrico $5,87 \pm 1,10$ vs $4,57 \pm 0,12 \mathrm{mg} / \mathrm{dL}(\mathrm{P}=0,02)$; creatinina $0,82 \pm 0,12$ vs $0,73 \pm 0,09 \mathrm{mg} / \mathrm{dL}(\mathrm{P}=0,01)$; peso do recém-nascido $2.130,7 \pm 839,3$ vs $3.555,0 \pm 261,0$ gramas $(\mathrm{P}=0.01)$ e peso da placenta $621,3 \pm 167,0$ vs $796,3 \pm 154,2$ gramas $(\mathrm{P}=0,05)$. A relação proteinúria/creatininúria no grupo das pacientes com pré-eclâmpsia foi de $2,40 \pm 1,31$. O valor de IL-6sR (ng/dL) na pré-eclâmpsia foi $28,7 \pm 10,8$ vs $16,5 \pm 6,4$ na gestante normotensa $(\mathrm{P}=0,01)$.

Conclusões: Estes resultados mostram o aumento dos níveis plasmáticos do IL-6sR em pacientes com pré-eclâmpsia, em relação a gestantes normotensas. Mais estudos se mostram necessários para o esclarecimento da fisiopatologia desta entidade, como a análise de outras citocinas ligadas a esse receptor, visto que elas podem ser a chave para a resposta inflamatória sistêmica que ocorre nestas pacientes e, portanto, para o seu tratamento.
\end{abstract}

DESCRITORES: PRÉ-ECLÂMPSIA; RECEPTORES DE INTERLEUCINA-6; INFLAMAÇÃO

\begin{abstract}
Aims: To compare Interleukin-6 soluble receptor (IL-6sR) plasmatic levels between normotensive pregnant controls and preeclamptic women.

Methods: Maternal blood samples were collected before delivery from 21 patients with preeclampsia and 39 normotensive pregnant controls. Samples were stored at $-80^{\circ} \mathrm{C}$ until laboratory assay. IL-6sR was measured by ELISA enzyme immunoassay. To compare groups Student's $t$ test was used. Results with P less than 0.05 were considered significant. Results: Data from preeclampsia and normotensive pregnant controls were respectively: maternal age $22.3 \pm 4.8 \mathrm{vS}$ $26.0 \pm 3.7$ years $(\mathrm{P}=0.06)$; gestational age $32.7 \pm 5.8$ vs $40.1 \pm 0.8$ weeks $(\mathrm{P}=0.01)$; systolic blood pressure $143.0 \pm 2.2$ vs $118.8 \pm 3.1 \mathrm{mmHg}(\mathrm{P}=0.01)$; diastolic blood pressure $112.5 \pm 4.0$ vs $77.2 \pm 10.2 \mathrm{mmHg}(\mathrm{P}=0.01)$; uric acid $5.87 \pm 1.10 v s$ $4.57 \pm 0.12 \mathrm{mg} / \mathrm{dL}(\mathrm{P}=0.02)$; creatinine $0.82 \pm 0.12$ vs $0.73 \pm 0.09 \mathrm{mg} / \mathrm{dL}(\mathrm{P}=0.01)$; birth weight $2130.7 \pm 839.3$ vs $3555.0 \pm 261.0 \mathrm{~g}(\mathrm{P}=0.01)$; placental weight $621.3 \pm 167.0$ vs $796.3 \pm 154.2 \mathrm{~g}(\mathrm{P}=0,05)$. Proteinuria over creatininuria ratio in the preeclampsia group was $2.40 \pm 1.31$. The concentration of IL-6sR (ng/dL) was $28.7 \pm 10.8$ in preeclampsia $v \mathrm{~s}$ $16.5 \pm 6.4$ in normotensive pregnant controls $(\mathrm{P}=0.01)$.

Conclusions: These results show an increased plasma levels of IL-6sR in patients with preeclampsia compared to normotensive pregnant women. More studies are necessary to clarify the pathophysiology of this entity, including the analysis of other cytokines linked to this receptor, due to the fact that they can be the key for the systemic inflammatory response that occurs in these patients and therefore for their treatment.
\end{abstract}

KEY WORDS: PREECLAMPSIA; RECEPTORS, INTERLEUKIN-6; INFLAMMATION. 


\section{INTRODUÇÃO}

A pré-eclâmpsia (PE) é uma síndrome específica da gravidez humana que ocorre após a vigésima semana de gestação e é caracterizada por hipertensão (níveis tensionais acima de $139 \mathrm{mmHg}$ de pressão sistólica e $89 \mathrm{mmHg}$ de pressão diastólica) e proteinúria patológica $(\geq 300 \mathrm{mg} / 24 \mathrm{~h})$, em mulheres previamente normotensas. ${ }^{1}$ Essa patologia é uma das principais causas de morte materna, sendo responsável por cerca de $3 \%$ a $8 \%$ da morbidade e mortalidade de gestantes em todo o mundo. ${ }^{2,3}$ Além disso, a PE aumenta a chance de parto prematuro, podendo, dessa forma, causar complicações perinatais. ${ }^{4,5,6}$

A etiologia da PE ainda não está bem estabelecida, entretanto muitos estudos têm apontado a placenta como a principal responsável pelas manifestações dessa condição, já que o parto é a única cura para a PE. ${ }^{7}$ Em contraste com uma gestação normal, na PE ocorre uma hipoperfusão uteroplacentária, podendo levar a hipóxia tecidual e isquemia placentária. Nesse processo, há liberação de diversos mediadores inflamatórios, além de marcantes alterações hemodinâmicas, responsáveis por um desequilíbrio entre fatores vasodilatadores e vasoconstritores, levando a um aumento da resistência vascular periférica e da pressão arterial sistêmica das gestantes. ${ }^{8}{ }^{89}$ Um dos principais mediadores inflamatórios envolvidos nesse processo é a Interleucina (IL)-6, um mediador sintetizado por fagócitos monononucleares, células endoteliais e fibroblastos em resposta a estímulos inflamatórios. IL-6 tem sido descrita alterada em mulheres com PE. ${ }^{10,11}$ Estudos experimentais também apresentaram alterações nessa citocina. ${ }^{12}$

Diferentes receptores são necessários para que a IL-6 realize sinalizações intracelulares: o receptor de IL-6 (IL-6R), expresso na membrana plasmática de células específicas, como hepatócitos e alguns leucócitos; $;^{13}$ uma forma solúvel desse receptor da IL-6, (IL-6sR), presente em fluidos corporais; e a proteína transmembrana transdutora de sinal, o gp130. IL-6sR forma um complexo solúvel com a citocina IL-6 (IL-6sR/IL-6) que se liga diretamente ao gp130 da membrana plasmática em células em que não há expressão do receptor de superfície IL-6R, em um processo conhecido como trans-sinalização. Esse processo permite que células inicialmente irresponsivas à IL-6 (pela falta do IL-6R ligado à membrana) respondam ao complexo que está presente em respostas inflamatórias. ${ }^{14}$ Está demonstrado também que o IL-6sR pode atuar como transportador da IL-6, aumentando a meia-vida dessa citocina, o que levaria o IL-6sR a aumentar a sinalização da IL-6, funcionando como um agonista da mesma. ${ }^{15}$
Visto que a PE está associada a uma exacerbação da resposta inflamatória, nossa hipótese é de que, assim como ocorre um aumento de mediadores inflamatórios como IL-6, a elevação dos níveis circulantes do seu receptor solúvel IL-6sR também deverá ocorrer. Desse modo, o objetivo deste trabalho é comparar os níveis plasmáticos de IL-6sR entre gestantes com PE e normotensas.

\section{MÉTODOS}

Foi conduzido um estudo transversal em gestantes que receberam atendimento obstétrico no Hospital São Lucas da Pontifícia Universidade Católica do Rio Grande do Sul (HSL/PUCRS). O projeto foi aprovado pelo Comitê de Ética em Pesquisa (CEP-671/11) e todas as gestantes consentiram em participar.

Foram analisadas coletas de 60 gestantes, sendo 21 com PE e 39 normotensas. A PE foi diagnosticada de acordo com as recomendações do National High Blood Pressure Education Program Working Group on High Blood Pressure in Pregnancy e das VI Diretrizes Brasileiras de Hipertensão. ${ }^{1,16}$ Pacientes com histórico de diabetes mellitus, doenças renais, hepáticas e infecciosas, polidrâmnio, gestação múltipla, ruptura prematura de membranas e normotensas com $1+$ ou mais de proteína no exame qualitativo de urina, foram excluídas. Proteinúria foi quantificada apenas em gestantes com PE.

No período pré-parto foram coletados $4 \mathrm{ml}$ de sangue materno em tubo Vacutainer ${ }^{\circledR}$ (Becton Dickinson \& Company, New Jersey, Estados Unidos) contendo ácido etileno diamino tetra-acético (EDTA). A amostra foi centrifugada a $1,000 \mathrm{~g}$ por 10 minutos, e o plasma armazenado a $-80^{\circ} \mathrm{C}$ até posterior análise laboratorial.

Parâmetros maternos e fetais, como idade materna, idade gestacional obstétrica, tipo de parto, raça, pressão arterial sistólica (PAS) e diastólica (PAD), peso materno, glicemia de jejum, índice de massa corpórea (IMC) ajustada à idade gestacional, ${ }^{17}$ peso do recém-nascido, peso da placenta, percentil de peso fetal e índice de Apgar no $1^{\circ}$ e no $5^{\circ}$ minuto, foram comparados entre as pacientes com PE e normotensas.

A concentração de IL-6sR no plasma materno foi mensurada através do teste imunoenzimático de ELISA (R\&D Systems Inc., Minneapolis, MN-USA), em duplicata. O ensaio foi validado para dosagem de plasma, alcançando um fator de diluição de 1:128 em reagente diluente. $\mathrm{O}$ coeficiente de variação intra e interensaio foi de $5-10 \%$, e $12 \%$, respectivamente.

O protocolo para avaliação da concentração de IL-6sR foi realizado conforme orientações do fabri- 
cante. Brevemente, placas de ELISA foram sensibilizadas com antígeno IL-6sR; as lavagens foram realizadas com Tween ${ }^{\circledR} 20$ 0,05\%; o bloqueio de sítios inespecíficos foi feito com albumina sérica bovina; a reação com o anticorpo de detecção foi seguida de estreptavidina. Para mensurar a intensidade de variação da absorbância foi utilizado espectrofotômetro (URIT-660 Microplate Reader, China) em comprimento de onda $450 \mathrm{~nm}$. Tendo em vista o fator de diluição, a concentração final de IL-6sR foi ajustada por cálculo.

A análise foi realizada através do programa Statistical Package for Social Sciences versão 17.0 (SPSS, 17.0) para Windows. As variáveis contínuas foram descritas por medidas de tendência central e dispersão (média e desvio-padrão). Nas comparações entre dois grupos foi empregado o teste $t$ de Student. As variáveis categóricas foram expressas em porcentagem e comparadas usando o teste Qui-quadrado. O nível de significância considerado foi de 0,05 .

\section{RESULTADOS}

Os dados clínicos e bioquímicos das pacientes e de seus neonatos estão apresentados na Tabela 1. Mulheres com PE apresentaram relação proteinúria/

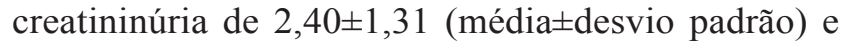
as pressões arterial sistólica e diastólica aumentadas

Tabela 1. Perfil demográfico e bioquímico das 60 gestantes incluídas no estudo e dados de seus neonatos.

\begin{tabular}{lccc}
\hline & $\begin{array}{c}\text { Pré-eclâmpsia } \\
(\mathbf{n = 2 1 )}\end{array}$ & $\begin{array}{c}\text { Normotensas } \\
(\mathbf{n = 3 9 )}\end{array}$ & $\mathbf{P}$ \\
\hline Idade média, anos & $22,3 \pm 4,8$ & $26,0 \pm 3,7$ & 0,06 \\
PAS, mmHg & $143,0 \pm 2,2$ & $118,8 \pm 3,1$ & 0,01 \\
PAD, mmHg & $112,5 \pm 4,0$ & $77,2 \pm 10,2$ & 0,01 \\
Ácido úrico, mg/dL & $5,87 \pm 1,10$ & $4,57 \pm 0,12$ & 0,02 \\
Creatinina, mg/dL & $0,82 \pm 0,12$ & $0,73 \pm 0,09$ & 0,01 \\
Idade gestacional, sem & $32,7 \pm 5,8$ & $40,1 \pm 0,8$ & 0,01 \\
Peso do & $2.130,7 \pm 839,3$ & $3.555,0 \pm 261,0$ & 0,01 \\
recém-nascido, g & $621,3 \pm 167,0$ & $796,3 \pm 154,2$ & 0,05 \\
Peso da placenta, g & $7,6 \pm 2,0$ & $7,6 \pm 2,4$ & 0,89 \\
Índice de Apgar, & & & \\
$1^{\circ}$ minuto & $9,1 \pm 0,9$ & $9,2 \pm 1,0$ & 0,59 \\
Índice de Apgar, & & & \\
$5^{\circ}$ minuto & & & 0,04 \\
Percentil de & & 73,3 & \\
peso fetal, \% & 66,7 & 26,7 & \\
AIG & 13,3 & 0 & \\
GIG & 20 & & \\
PIG & & & \\
\hline
\end{tabular}

PAS: pressão arterial sistólica; PAD: pressão arterial diastólica; n: púmero de casos. AIG: adequado para a idade gestacional; GIG: grande para a idade gestacional; PIG: pequeno para a idade gestacional. Os valores foram apresentados em média \pm desvio padrão; teste $t$ de Student. Os valores de percentil de peso fetal estão expressos em porcentagem; teste Qui-quadrado.
$(\mathrm{P}<0,05)$. Da mesma forma, os níveis de ácido úrico e creatinina foram significativamente maiores nas pacientes com $\mathrm{PE}$ em relação às gestantes normotensas $(\mathrm{P}<0,05)$. A idade gestacional e o peso do recém-nascido foram menores em pacientes do grupo $\mathrm{PE}(\mathrm{P}<0,05)$. Observou-se uma maior proporção de recém-nascidos pequenos para a idade gestacional em pacientes com $\mathrm{PE}$, e uma maior proporção de grandes para a idade gestacional em gestantes normotensas $(\mathrm{P}<0,05)$. Tanto a idade materna quanto o peso da placenta foram equivalentes nos grupos estudados.

Os valores de IL-6sR (ng/dL) no grupo PE foram mais elevados do que nas gestantes normotensas, com resultados de $28,7 \pm 10,8$ na $\mathrm{PE}$ vs $16,5 \pm 6,4$ (média \pm desvio padrão, $\mathrm{P}=0,01$ ), como demonstrado na Figura 1.

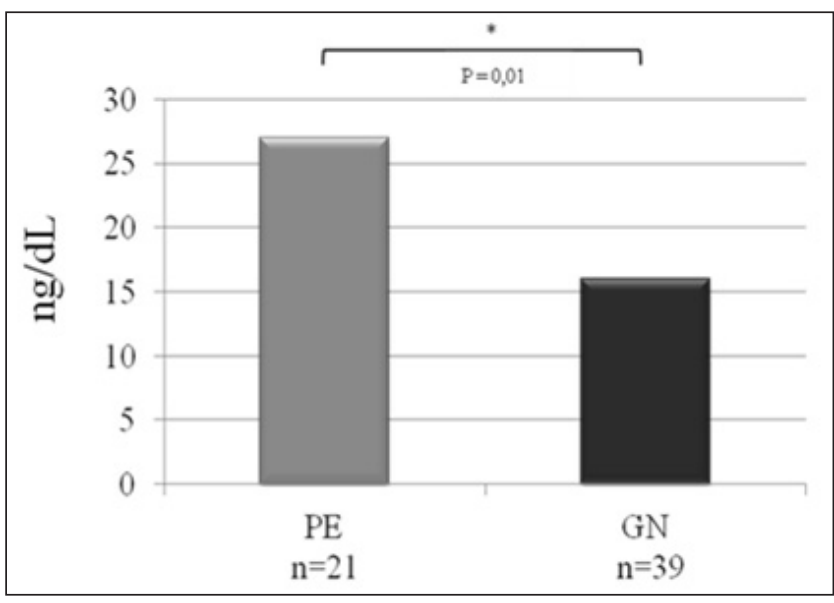

Figura 1. Médias dos níveis de receptor solúvel da interleucina-6 (IL-6sR) nas gestantes com pré-eclâmpsia (PE) $(28,7 \pm 10,8)$ e normotensas (GN) $(16,5 \pm 6,4)$.

\section{DISCUSSÃO}

O presente trabalho apresenta a elevação dos níveis plasmáticos de IL-6sR - o qual forma um complexo ligante-receptor com IL-6, estimulando a proliferação, diferenciação e processos inflamatórios - em amostra de pacientes com PE comparadas às normotensas, no terceiro trimestre gestacional.

Os resultados clínicos obtidos no presente estudo estão de acordo com os esperados nessa patologia. $\mathrm{O}$ baixo peso ao nascer dos neonatos de gestantes com PE em relação ao de gestantes normotensas pode ser parcialmente explicado pela tendência à interrupção precoce da gestação observada nessa patologia. Este fato é comprovado pela diferença de idade gestacional no momento do parto entre os dois grupos. A precocidade do parto também pode ocorrer devido à 
disfunção placentária que ocorre na PE, acarretando um importante papel no baixo peso dos recém-nascidos, assim como no peso da placenta. ${ }^{18,19}$

Valores de pressão arterial sistólica e diastólica aumentados em pacientes com PE corroboram com o diagnóstico desse distúrbio, o mesmo valendo para níveis de proteinúria, ácido úrico e creatinina, sendo estes achados esperados e, portanto, de acordo com a literatura. ${ }^{1}$

As citocinas estão envolvidas na fisiologia de uma gestação normal, desde a implantação do concepto no útero, até o final do período gravídico, passando por todas as adaptações do desenvolvimento fetal na vida intrauterina. ${ }^{20} \mathrm{O}$ sistema imune adaptativo envolve ações de células T, B e células Natural Killer. As células $\mathrm{T}$ podem ser divididas em $\mathrm{T}$ helper $(\mathrm{Th})$ ou $\mathrm{T}$ auxiliar (Ta) expressando CD4+, e T citotóxicas expressando CD8+. As células T CD4+ podem ser subdivididas em duas populações efetoras distintas: Th1 e Th2, as quais produzem respectivamente INF- $\gamma$, IL- 2 , TNF- $\beta$ e TNF- $\alpha$ essenciais para o estabelecimento de respostas imunes celulares, e IL-4, IL-5, IL-6, IL-10 e IL-13 que são importantes na formação de anticorpos, reações imunes mediadas por eosinófilos e mastócitos. ${ }^{21,22,23}$ $\mathrm{Na}$ gestação normal há supressão de Th1 em relação a Th2 para o desenvolvimento fetal, já na PE há um desbalanço nessa relação, não havendo supressão adequada em Th1.21

Níveis circulantes de Fator de Necrose Tumoral (TNF)-alfa e IL-6, normalmente aumentados em gestações normais, apresentam-se ainda mais elevados em pacientes com PE. ${ }^{20} \mathrm{Da}$ mesma forma, várias outras citocinas e moduladores, como IL-2, IL-8, IL-12, IL-15, IL-18, IL-1R, receptor solúvel de IL-4 e receptor solúvel de TNF, podem ser vistos em grande quantidade nessa síndrome. Os relatos do aumento de citocinas pró-inflamatórias em pacientes com PE sugere uma exacerbação da resposta pró-inflamatória nessas gestantes. ${ }^{1,24,25}$

A IL-6 mostrou estar associada à exacerbação inflamatória observada nesse distúrbio hipertensivo gestacional. ${ }^{12,24,26,27}$ No entanto, parece existir ainda uma dúvida em relação ao seu papel na PE, visto que em um trabalho analisando soro materno e fetal e o tecido placentário de gestantes com PE e normotensas, esta associação não foi encontrada. ${ }^{28}$

O complexo IL-6R, IL-6sR, gp130 é necessário à recepção da mensagem de IL-6, entretanto $70 \%$ da IL-6 que circula no sangue está ligada somente ao seu receptor solúvel. Esse complexo IL-6Rs/IL-6 liga-se diretamente ao gp130 da membrana plasmática realizando a trans-sinalização que, a partir de sua dimerização, inicia a sinalização intercelular pela via JAK/STAT. ${ }^{14,29}$ Os outros $30 \%$ da IL-6 ligam-se diretamente ao receptor de membrana IL-6R..$^{30}$ Acredita-se que a gp130 está expressa em todas as células do corpo, enquanto o IL-6R é expresso principalmente em hepatócitos, neutrófilos, monócitos/ macrófagos e alguns linfócitos. Além das moléculas citadas, a expressão do supressor de sinalização de citocina (SOCS) também tem um papel importante na via da IL-6. As SOCS são proteínas induzidas pela via JAK/STAT e inibem a transdução do sinal de STAT, funcionando, portanto como inibidoras por feedback. Sabe-se que a SOCS3 pode inibir potencialmente a sinalização da citocina IL-6. ${ }^{31}$

Além da IL-6sR, a gp130 também tem sua forma solúvel, conhecida como sgp130. A sgp130 pode se ligar ao complexo solúvel IL-6sR/IL-6 impedindo que este se vincule ao gp130 da membrana plasmática da célula-alvo, funcionando assim como um antagonista do efeito da IL-6. ${ }^{32}$ Essas moléculas estão descritas como alteradas na placenta de mulheres com PE. ${ }^{33}$ Através de investigações in vitro, constatou-se que o IL-6sR contribui para a ativação de uma variedade de processos celulares. No entanto, a compreensão da importância funcional deste receptor in vivo ainda é incipiente. ${ }^{34,35}$ Diversas condições, como câncer de próstata e mama, artrite reumatoide, peritonite e esclerose múltipla apresentaram fisiopatologias relacionadas ao IL-6sR até o momento, o qual, associado à IL-6, sugere um importante papel na regulação do sistema imune. ${ }^{34,36,37,38,39}$ Isso parece ocorrer devido à sua propriedade solúvel, podendo circular em diversas células do corpo, diferentemente do IL-6R, que é expresso exclusivamente na membrana plasmática de algumas células. ${ }^{40}$ No entanto, devido à natureza pleiotrópica da IL-6, que, além de efeitos próinflamatórios, já teve diversos outros efeitos atribuídos, inclusive de imunossupressão, a compreensão dos efeitos clínicos da IL-6 na PE torna-se complexa. ${ }^{24,41}$

Por fim, esta pesquisa está de acordo com estudos recentes que enfatizaram as propriedades pró-inflamatórias do IL-6sR mediante associação com IL-6 e gp-130. Resultados avaliando somente o IL-6sR, como visto neste trabalho, contribuem para o conhecimento da participação dessas moléculas na PE. Nossos resultados mostram o aumento dos níveis plasmáticos do IL-6sR em pacientes com PE, em relação a gestantes normotensas. Mais estudos se mostram necessários para o esclarecimento da fisiopatologia desta entidade, como a análise de outras citocinas ligadas a esse receptor, visto que elas podem ser a chave para a resposta inflamatória sistêmica que ocorre nestas pacientes e, portanto, para o seu tratamento. 


\section{AGRADECIMENTOS}

O Laboratório de Nefrologia da PUCRS recebe apoio de CAPES, FAPERGS, CNPq e PUCRS. Hentschke MR é bolsista da CAPES e Poli-deFigueiredo CE é pesquisador do CNPq.

\section{REFERENCIAS}

1. Report of the National High Blood Pressure Education Program Working Group on High Blood Pressure in Pregnancy. Am J Obstet Gynecol. 2000;183(1):S1-S22.

2. Duley L. The global impact of pre-eclampsia and eclampsia. Semin Perinatol. 2009;33:130-7.

3. Campbell KH, Savitz D, Werner EF, Pettker CM, Goffman D, Chazotte C, Lipkind HS. Maternal Morbidity and Risk of Death at Delivery Hospitalization. Obstet Gynecol. 2013;122(3):627-33.doi: 10.1097/AOG.0b013e3182a06f4e

4. Zhang YP, Liu XH, Gao SH, Wang JM, Gu YS, Zhang JY, Zhou X, Li QX. Risk factors for preterm birth in five Maternal and Child Health hospitals in Beijing. PloS one. 2012;7(12):e52780.

5. Gagliardi L, Rusconi F, Da Frè M, Mello G, Carnielli V, Di Lallo D, Macagno F, Miniaci S, Corchia C, Cuttini M. Pregnancy disorders leading to very preterm birth influence neonatal outcomes: results of the population-based ACTION cohort study. Pediatr Res. 2013;73(6):794-801.

6. Rana S, Schnettler WT, Powe C, Wenger J, Salahuddin S, Cerdeira AS, Verlohren S, Perschel FH, Arany Z, Lim KH, Thadhani R, Karumanchi SA. Clinical characterization and outcomes of preeclampsia with normal angiogenic profile. Hypertens Pregnancy. 2013;32(2):189-201.

7. Chesley LC. Hypertension in pregnancy: definitions, familial factor and remote prognosis. Kidney Int 1980;18(2):234-40.

8. Gilbert JS, Ryan MJ, LaMarca BB, Sedeek M, Murphy SR, Granger JP. Pathophysiology of hypertension during preeclampsia: linking placental ischemia with endothelial dysfunction. Am J Physiol Heart Circ Physiol. 2008;294(2):H541-50.

9. Roberts JM, Hubel CA. The two stage model of preeclampsia: variations on the theme. Placenta. 2009;30:S32-7.

10. Jonsson Y, Rubèr M, Matthiesen L, Berg G, Nieminen K, Sharma S, Ernerudh J, Ekerfelt C.Cytokine mapping of sera from women with preeclampsia and normal pregnancies. J Reprod Immunol. 2006;70(1-2):83-91.

11. Takacs P, Grenn KL, Nikaeo A, Kauma SW. Increased vascular endothelial cell production of interleukin- 6 in severe preeclampsia. Am J Obstet Gynecol. 2003;188(3):740-4.

12. Gadonski G, LaMarca BBD, Sullivan E, Bennett W, Chandler D, Granger JP. Hypertension Produced by Reductions in Uterine Perfusion in the Pregnant Rat: Role of Interleukin 6. Hypertens. 2006;48(4):711-6.

13. Rose-John S. IL-6 trans-signaling via the soluble IL-6 receptor: importance for the pro-inflammatory activities of IL-6. Int J Biol Sci. 2012;8(9):1237-47.

14. Knüpfer H, Preiss. R. sIL-6R: more than an agonist? Immunol Cell Biol. 2008,86(1):87-91.

15. Peters M, Jacobs S, Ehlers M, Vollmer P, Mullberg J, Wolf E, Brem G, Meyer zumBüschenfelde KH, Rose-John S. The function of the soluble interleukin 6 (IL-6) receptor in vivo: sensitization of human soluble IL-6 receptor transgenic mice towards IL-6 and prolongation of the plasma half-life of IL- 6. J Exp Med.1996;183(4):1399-406.
16. Sociedade Brasilerira de Cardiologia, Sociedade Brasileira de Hipertensão, Sociedade Brasileira de Nefrologia. VI Brazilian Guidelines on Hypertension. Arq Bras Cardiol. 2010;95(1 Suppl):1-51.

17. Atalah E, Castillo C, Castro R, Aldea A. Proposal of a new standard for the nutriotional assessment of pregnant women. Rev Med Chil. 1997;125(12):1429-36.

18. Hentschke MR, Poli-de-Figueiredo CE, Costa BEP, Kurlak LO, Williams PJ, Mistry HD. Is the atherosclerotic phenotype of preeclamptic placentas due to altered lipoprotein concentrations and placental lipoprotein receptors? Role of a small for gestational age phenotype. J Lipid Res. 2013;54(10):2658-64. doi:10.1194/jlr.M036699

19. Kadyrov M, Moser G, Rath W, Kweider N, Wruck CJ, Pufe T, Huppertz B. Maternal-placental interactions and fetal programming. Z GeburtshilfeNeonatol. 2013;217(3):88-94. doi: 10.1055/s-0033-1347179. 2013

20. Rusterholz C, Hahn S, Holzgreve W. Role of placentally produced inflammatory and regulatory cytokines in pregnancy and the etiology of preeclampsia. Semin Immunopathol. 2007;29(2):151-62.

21. Sargent IL, Borzychowski AM, Redman CW. Immuno regulation in normal pregnancy and pre-eclampsia: an overview. Reprod Biomed Online. 2007;14 Spec No 1: 111-7. doi: 10.1016/S1472-6483(10)61465-4

22. Erlebacher A. Immunology of the maternal-fetal interface. Annu Rev Immunol. 2013;31:387-411. doi: 10.1146/annurevimmunol-032712-100003

23. Abbas AK, Lichtman AH, Pillai S. Imunologia celular e molecular. $7^{\mathrm{a}}$ ed. Rio de Janeiro: Elsevier Brasil; 2012.

24. Bernardi F, Guolo F, Bortolin T, Petronilho F, Dal-Pizzol F. Oxidative stress and inflammatory markers in normal pregnancy and preeclampsia. J ObstetGynaecol Res. 2008; 34(6):948-51.

25. Schiessl B. Inflammatory response in preeclampsia. Molecular Aspects of Medicine. 2007;28(2):210-9.

26. Zhang Z, Gao Y, Zhang L, Jia L, Wang P, Li H. Alterations of IL-6, IL-6R and gp130 in early and late onset severe preeclampsia. Hypertens Pregnancy. 2013;32(3):270-80. doi:10.3109/10641955.2013.798332

27. Guven MA, Coskun A, Ertas IE, Aral M, Zencirci B, Oksuz H. Association of maternal serum CRP, IL-6, TNF-alpha, homocysteine, folic acid and vitamin B12 levels with the severity of preeclampsia and fetal birth weight. Hypertens Pregnancy. 2009;28(2):190-200.

28. Al-Othman S, Omu AE, Diejomaoh FM, Al-Yatama M, AlQattan F. Differential levels of interleukin 6 in maternal and cord sera and placenta in women with pre-eclampsia. Gynecol Obstet Invest. 2001;52(1):60-5.

29. Taga T, Kishimoto T. Gp130 and the interleukin-6 family of cytokines. Annu Rev Immunol. 1997;15:797-819.

30. Gaillard J, Pugniere M, Tresca J, Mani J, Klein B, Brochier J. Interleukin-6 receptor signaling. II. Bio-availability of interleukin-6 in serum. Eur Cytokine Netw. 1999;10(3): 337-44.

31. Heinrich PC, Behrmann I, Haan S, Hermanns HM, MüllerNewen G, Schaper F. Principles of interleukin (IL)-6-type cytokine signaling and its regulation. Biochem J.2003;374 (Pt 1):1-20.

32. Jostock T, Müllberg J, Ozbek S, Atreya R, Blinn G, Voltz N, Fischer M, Neurath MF, Rose-John S. Soluble gp130 is the natural inhibitor of soluble interleukin-6 receptor transsignaling responses. Eur J Biochem. 2001;268(1): $160-7$. 
33. Zhao S, Gu Y, Dong Q, Fan R, Wang Y.Altered interleukin-6 receptor, IL-6R and gp130, production and expression and decreased SOCS-3 expression in placentas from women with pre-eclampsia.Placenta. 2008;29(12):1024-8. doi: 10.1016/ j.placenta.2008.09.011

34. Hurst SM, Wilkinson TS, McLoughlin RM, Jones S, Horiuchi S, Yamamoto N, Rose-John S, Fuller GM, Topley N, Jones SA. Il-6 and its soluble receptor orchestrate a temporal switch in the pattern of leukocyte recruitment seen during acute inflammation. Immunity. 2001;14(6):705-14.

35. Jones SA and Rose-John S. The role of soluble receptors in cytokine biology: the agonistic properties of the sIL-6R/IL-6 complex. Biochim Biophys Acta. 2002;1592(3):251-63.

36. Shariat SF, Kattan MW, Traxel E, Andrews B, Zhu K, Wheeler TM, Slawin KM. Association of pre- and postoperative plasma levels of transforming growth factor beta(1) and interleukin 6 and its soluble receptor with prostate cancer progression. Clin Cancer Res. 2004;10(6):1992-9.

37. Singh A, Purohit A, Wang DY, Duncan LJ, Ghilchik MW, Reed MJ. IL-6sR: release from MCF-7 breast cancer cells and role in regulating peripheral oestrogen synthesis. J Endocrinol. 1995;147(2):R9-12.

38. Nishimoto N, Terao K, Mima T, Nakahara H, Takagi N, Kakehi T. Mechanisms and pathologic significances in increase in serum interleukin-6 (IL-6) and soluble IL-6 receptor after administration of an anti-IL-6 receptor antibody, tocilizumab, in patients with rheumatoid arthritis and Castleman disease. Blood. 2008;112(10):3959-64.

39. Tilbery CR, Felipe E, Mota IM, Scheinberg MA. Aumento dos receptores solúveis da interleucina-2 na esclerose múltipla. Arq Neuropsiquiatr. 1994;52(2):216-20.

40. Rose-John S, Heinrich PC. Soluble receptors for cytokines and growth factors: generation and biological function. Biochem. J. 1994;300(Pt 2):281-90.

41. Lau SY, Guild SJ, Barrett CJ, Chen Q, McCowan L, Jordan V, Chamley LW. Tumor Necrosis Factor-Alpha, Interleukin-6, and Interleukin-10 Levels are Altered in Preeclampsia: A Systematic Review and Meta-Analysis. Am J Reprod Immunol. 2013;70(5):412-27. doi: 10.1111/ aji.12138. 